Bull. Austral. Math. Soc.

20D15, 20-04

Vol. 59 (1999) [343-346]

\title{
ON THE SOLUBLE LENGTH OF GROUPS WITH PRIME-POWER ORDER
}

\author{
Susan Evans-Riley, M.F. Newman and Csaba Schneider
}

To B. H. Neumann in his 90th year.

We show that for every integer $k \geqslant 3$ and every prime $p \geqslant 5$ there is a group with soluble length $k$ and order $p^{2^{k}-2}$.

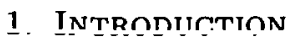

There has been interest since the time of Burnside in the question: given a prime $p$ and a positive integer $k$ what is the smallest order of a group of $p$-power order with soluble length (exactly) $k$ ? Let $p^{\beta_{p}(k)}$ denote this smallest order.

The first paper which discussed problems like this is one by Burnside [4] in 1913. In that paper he observed that there are groups of order $p^{3}$ with soluble length 2; and groups of order $p^{6}$ with soluble length 3 . Moreover he showed that a group with soluble length $k+1$ must have order at least $p^{3 k}$; and said: but it seems probable that for greater values of $k$ the actual lower limit for the order exceeds $p^{3 k}$.

Burnside [5] confirmed this expectation by proving: $p^{(k+1)(k+2) / 2}$ is a lower limit for the order of a prime power group whose $k$-th derived group is not the identity; and moreover: this lower limit is not attained except when $k$ is 1 or 2 . So, in particular, a $p$-group with soluble length 4 has order at least $p^{11}$.

The theme was taken up and a major advance was made by $\mathrm{P}$. Hall and reported in his now famous paper [8] of 1934. In it he proved that $2^{k-1}+k-1 \leqslant \beta_{p}(k) \leqslant 2^{k-2}\left(2^{k-1}-1\right)$ (pp. 56-7). For $k \leqslant 4$ this lower bound is no better than Burnside's bound. Hall also established that $\beta_{2}(k) \leqslant 2^{k}-1$.

In 1950 Itô [12] refined the upper bound given by Hall. He showed that $\beta_{p}(k) \leqslant$ $3 \cdot 2^{k-1}$. Further progress was made by Blackburn in his thesis [1] in 1956. [The relevant part of that work has never been otherwise published or announced. We are indebted to Professor Blackburn for recently supplying us with a copy of his thesis.] Blackburn

Received 15th October, 1998

We are indebted to Professor Avinoam Mann for drawing our attention to his unpublished result that $2^{k-1}+2 k-4 \leqslant \beta_{p}(k)$ and to Blackburn's thesis. We are also indebted to Dr Werner Nickel for his helpful comments on a draft.

Copyright Clearance Centre, Inc. Serial-fee code: 0004-9729/99 \$A2.00+0.00. 
proved [1, Theorem 10] for every $p>3$ every group of order $p^{13}$ has soluble length at most 3 ; therefore $\beta_{p}(4) \geqslant 14$. He also described examples of Hall, later published in [9], of groups with order $p^{2^{k}-1}$ and soluble length $k$ (for $p$ odd). (Details of the Hall examples can be found conveniently in Huppert [11, Satz III.17.7].)

In this paper we present examples which complete the story for $k=4$. Namely, there are $p$-groups with soluble length 4 and order $p^{14}$ for all $p \geqslant 5$. More generally, in Section 3 we describe a series of finite $p$-groups with soluble length $k$ and order $p^{2^{k}-2}$ for all $p \geqslant 5$. In the context of this problem the primes 2,3 behave differently already for soluble length 3 (see Blackburn [2, pp. 89-91]).

One of the contributions that Burnside and Hall made was to show the significance of commutators in the study of groups of prime-power order. Hence some results about p-groups come as corollaries of results about nilpotent groups. In Section 2 we show that there is a 2-generator torsion-free nilpotent group with soluble length 4 and Hirsch length 14. (The Hirsch length is the number of infinite cyclic factors in a polycyclic series.) This group has nilpotency class 11 . Factoring out the $p$-th powers of the generators gives that for all $p \geqslant 11$ there is $p$-group with soluble length 4 , order $p^{14}$ and exponent $p$. Examples for the primes 5 and 7 are also described in Section 2.

While, in some sense, the upper and lower bounds have the same order of magnitude, there is still a gap to fill even at this level. The quoted results give $k-1<\log _{2} \beta_{p}(k)<k$. Does $\log _{2} \beta_{p}(k)-k$ have a limit and, if so, what is it?

\section{Soluble length 4}

Consider the pro-p-presentation

$$
\begin{aligned}
\{a, b \mid & a^{p}=1, b^{p}=1,[b, a, b]=1,[b, a, a, a, a, a]=1 \\
& {[b, a, a, a, b, a, a, b, a, a, a]=1\} . }
\end{aligned}
$$

With the help of the $p$-Quotient Program (Havas et al. [10]) it is easy to establish that the pro-p-group $G_{p}$ defined by this presentation is a finite $p$-group of order $p^{14}$ for $p=5,7,11-$ and other individual primes as far as resources allow. Moreover, using the same program, one can see that the presentation

$$
\begin{aligned}
\{a, b \mid & a^{p}=1, b^{p}=1,[b, a, b]=1,[b, a, a, a, a, a]=1, \\
& {[b, a, a, a, b, a, a, b, a, a, a]=1, } \\
& {[[[b, a, a, a],[b, a]],[[b, a, a],[b, a]]]=1\} }
\end{aligned}
$$

defines a finite $p$-group of order $p^{13}$ for the same primes. Thus $G_{p}$ must have soluble length at least 4.

A more general result can be obtained by using the Nilpotent Quotient Program (Nickel [14]) as follows. Let $G$ denote the group defined by the presentation

$$
\{a, b \mid[b, a, b]=1,[b, a, a, a, a, a]=1,[b, a, a, a, b, a, a, b, a, a, a]=1\} .
$$


Then the program shows that the largest class 11 quotient of $G$ has Hirsch length 14 . Moreover the largest class 11 quotient of the group $H$ defined by the presentation

$$
\begin{aligned}
\{a, b \mid & {[b, a, b]=1,[b, a, a, a, a, a]=1, } \\
& {[b, a, a, a, b, a, a, b, a, a, a]=1, } \\
& {[[[b, a, a, a],[b, a]],[[b, a, a],[b, a]]=1\} }
\end{aligned}
$$

has Hirsch length 13 .

Hence the largest torsion-free and class 11 quotient of $G$ has Hirsch length 14 and soluble length 4.

The presentations used in this section were suggested by some in Caranti et al. [6] and the considerations in the next section.

\section{THE general CASE}

Our starting point is the $p$-adic Lie algebra $\mathcal{T}$ of dimension 8 described in Caranti et al. [6]. Note that $\mathcal{T}$ is a free $\mathbb{Z}_{p}$-module with $\mathbb{Z}_{p}$-basis $\{x, y, c, d, v, s, t, w\}$ and the multiplication can be described by the following table:

$$
\begin{array}{lllll}
{[y, x]=c,} & {[c, x]=d,} & {[c, y]=0,} & {[d, x]=v,} & {[d, y]=0} \\
{[v, x]=s,} & {[v, y]=t,} & {[s, x]=0,} & {[s, y]=2 w,} & {[t, x]=w} \\
& {[t, y]=0,} & {[w, x]=p x,} & {[w, y]=-2 p y} &
\end{array}
$$

It is shown in Caranti et al. that the quotients $\mathcal{T}_{j} / \mathcal{T}_{j+1}$ of the lower central series have characteristic $p$. The dimensions (over the field of $p$ elements) of the $\mathcal{T}_{j} / \mathcal{T}_{j+1}$ are periodic with period $2,1,1,1,2,1$. The terms $\mathcal{D}_{k}$ of the derived series are easy to calculate and this gives that $\mathcal{T}_{j+1}<\mathcal{D}_{k}<\mathcal{T}_{j}$, for $k \geqslant 2$, where $j=3 \cdot 2^{k-1}-1$. (Thus $\mathcal{T}_{j} / \mathcal{T}_{j+1}$ is the second 2-dimensional lower central factor of the appropriate period.) This means that $\mathcal{D}_{k}$ is one of the $p+1$ ideals between $\mathcal{T}_{j+1}$ and $\mathcal{T}_{j}$. Factoring out one of the $p$ other ideals gives an algebra of class $j$, soluble length $k+1$ and dimension $2^{k+1}-2$. The same $p$-adic algebra appears in Klaas et al. [13, p. 51] (taking $\Pi=\sqrt{p / 3}$ ). Using the Cayley map $x \mapsto(1-x)(1+x)^{-1}$ whose properties are described in [13, pp. 31-7] gives a pro-p-group with the corresponding lattice of normal subgroups. The corresponding quotient is a group with soluble length $k+1$ and order $p^{2^{k+1}-2}$.

\section{REFERENCES}

[1] N. Blackburn, Problems on the theory of finite groups of prime-power order, (Ph.D. Thesis) (University of Cambridge, Cambridge, U.K., 1956).

[2] N. Blackburn, 'On a special class of p-groups', Acta Math. 100 (1958), 45-92.

[3] W. Bosma, J. Cannon and C. Playoust, 'The Magma algebra system I: The user language', J. Symbolic Comput. 24 (1997), 235-265. 
[4] W. Burnside, 'On some properties of groups whose orders are powers of primes', Proc. London Math. Soc. (2) 11 (1913), 225-243.

[5] W. Burnside, 'On some properties of groups whose orders are powers of primes', (second paper), Proc. London Math. Soc. (2) 13 (1914), 6-12.

[6] A. Caranti, S. Mattarei, M.F. Newman and C.M. Scoppola, 'Thin groups of prime-power order and thin Lie algebras', Quart. J. Math. Oxford (2) 47 (1996), 279-296.

[7] K.W. Gruenberg and J.E. Roseblade (eds), The collected works of Philip Hall (Clarendon Press, Oxford, 1988).

[8] P. Hall, 'A contribution to the theory of groups of prime-power order', Proc. London Math. Soc. (2) 36 (1934), 29-95; or collected works [7] pp. 57-125.

[9] P. Hall, 'A note on $\overline{S I}$-groups', J. London Math. Soc. 39 (1964), 338-344; or collected works [7] pp. 653-661.

[10] G. Havas, M.F. Newman and E.A. O'Brien, 'The ANU p-Quotient Program (version 1.4)', The Australian National University, Canberra, (1996); Available as part of GAP [15] and MAGMA [3]; also via ftp from ftpmaths.anu.edu.au/pub/algebra/PQ/.

[11] B. Huppert, Endliche Gruppen I, Die Grundlehren der Mathematischen Wissenschaften 134 (Springer-Verlag, Berlin, Heidelberg, New York, 1967).

[12] N. Itô, 'Note on p-groups', Nagoya Math. J. 1 (1950), 113-116.

[13] G. Klaas, C.R. Leedham-Green and W. Plesken, Linear pro-p-groups of finite width, Lecture Notes in Mathematics 1674 (Springer-Verlag, Berlin, Heidelberg, New York, 1997).

[14] W. Nickel, 'The ANU Nilpotent Quotient Program (version 1.2)', Available as part of GAP [15]; also via ftp from ftpmaths.anu.edu.au/pub/algebra/NQ/.

[15] M. Schönert et al., GAP - Groups, Algorithms and Programming, (fifth edition) (Lehrstuhl D für Mathematik, Rheinisch Westfälische Technische Hochschule, Aachen, Germany, 1995).

School of Mathematics and Statistics

The University of Sydney

New South Wales 2006

Australia

e-mail: susan@maths.usyd.edu.au

School of Mathematical Sciences

The Australian National University

Canberra ACT 0200

Australia

e-mail: csaba@maths.anu.edu.au

\author{
School of Mathematical Sciences \\ The Australian National University \\ Canberra ACT 0200 \\ Australia \\ e-mail: newman@maths.anu.edu.au
}

